\title{
Office and 24-h ambulatory blood pressure control by treatment in general practice: the 'Monitoraggio della pressione ARteriosa nella medicina TErritoriale' study Augusto Zaninelli ${ }^{a}$, Gianfranco Parati ${ }^{b}$, Claudio Cricelli ${ }^{c}$, Angelo A. Bignamini ${ }^{d}$, Pietro A. Modesti ${ }^{a}$, Franco Pamparana ${ }^{\mathrm{e}}$, Grzegorz Bilo ${ }^{\mathrm{b}}$, Giuseppe Mancia ${ }^{\mathrm{b}}$ and Gian F. Gensini ${ }^{\mathrm{a}}$, On behalf of MARTE Investigators
}

Background Guidelines recommend that blood pressure (BP) should be lowered in hypertensive patients to prevent cardiovascular accidents. Management of antihypertensive treatment by general practitioners is usually based on office measurements, which may not allow an assessment of BP control over $24 \mathrm{~h}$, which requires ambulatory BP monitoring (ABPM) to be implemented. This is rarely done in general practice, and limited information is available on the consistency between the evaluations of the response to treatment provided by office measurement and by ABPM in this setting.

Aim To assess concordance between office BP measurements and ABPM-based estimates of hypertension control in a general practice setting.

Design of study Prospective, comparative between techniques.

Setting General practice.

Methods Seventy-eight general practices, representative of all Italian regions, participated in this study by recruiting sequential hypertensive adults on stabilized treatment, who were subdivided into even groups with office BP, respectively, controlled or noncontrolled by treatment. In each individual, ABPM was applied by the general practitioner after appropriate training, and 24-h ABP values were defined as controlled or not according to current guidelines. Concordance between office and ABPM evaluation of BP control was assessed with $\kappa$ statistics. Positive and negative predictive values of office measurement versus ABPM were estimated.

Results Between July 2005 and November 2006, 190 general practitioners recruited 2059 hypertensive patients based on office BP measurements; in 1728 patients, a 24-h

\section{Introduction}

Current hypertension guidelines recommend blood pressure (BP) to be satisfactorily 'controlled' to achieve cardiovascular protection, both in primary and secondary prevention. This implies the achievement of the identified BP targets, currently set at levels below 140/ $90 \mathrm{mmHg}$ in the office and below $125 / 80 \mathrm{mmHg}$ over
ABPM was performed, yielding 1524 recordings considered as valid for further analysis. The agreement between the assessment of BP control by office measurement and by ABPM was poor $(\kappa=0.120)$, with office measurements showing a satisfactory positive predictive value $(0.842)$ and a poor negative predictive value $(0.278)$; the situation was worse in patients with three or more among the following features: male sex, age of at least 65 years, alcohol consumption, diabetes, and obesity (negative predictive value $=0.149$ ) .

Conclusion In general practice, the agreement between assessment of BP control by treatment provided by office and ambulatory BP measurements is better in patients of 'uncontrolled' office BP than in 'controlled' office BP patients. This emphasizes the need for the larger use of outof-office BP monitoring in a general practice setting, in particular, in patients considered as 'controlled' during consultation. J Hypertens 28:910-917 @ 2010 Wolters Kluwer Health | Lippincott Williams \& Wilkins.

Journal of Hypertension 2010, 28:910-917

Keywords: blood pressure determination, blood pressure monitoring, hypertension

Abbreviations: ABPM, ambulatory BP monitoring; BP, blood pressure; NPV, negative predictive value; PPV, positive predictive value

aschool of Medicine, University of Florence, Florence, ' University of MilanoBicocca, Milan \& S.Luca Hospital, Istituto Auxologico Italiano, Milan, 'Italian College of General Practitioners (SIMG), Florence, d School of Specialization in Hospital Pharmacy, University of Milan and ${ }^{\mathrm{e}}$ Medical Department, Bayer Healthcare, Milan, Italy

Correspondence to Professor Augusto Zaninelli, MD, FAHA, General Practice, School of Medicine, University of Florence, via Marconi 15, 24058 Romano di Lombardia, Florence, Italy

Tel: +39 03358044265; fax: +39 0363901170; e-mail: zaninelli@interac.it

Received 8 December 2009 Accepted 11 January 2010

the $24 \mathrm{~h}[1-3]$. General practitioners are expected to play a major role in the attempt to achieve BP normalization in hypertensive patients, but the results of their intervention are often reported as unsuccessful, at least in Italy [3,4]. Furthermore, focus of general practitioners is, in most cases, only on office BP measurements to estimate the degree of hypertension control [5], 
regardless of the increasing evidence on the advantages carried by use of 24-h ambulatory BP monitoring $(\mathrm{ABPM})$ in this context $[6,7]$ and of the reports on the different BP thresholds to be considered with this approach in relation to cardiovascular risk [8-11]. Only a limited proportion of hypertensive patients, however, are managed also by considering the information carried by ABPM, which is usually applied and interpreted by cardiologists without the direct involvement of the general practitioner [5].

Only a few studies [12-14] have addressed the concordance between office and ABPM assessment of hypertension control, focusing in most cases on the evaluation of how often patients considered not controlled by the general practitioner were instead controlled according to ABPM, with the aim being to reduce the costs of hypertension management. This perspective is based on the hypothesis that office BP measurements may overestimate uncontrolled hypertension due to a possible 'whitecoat effect' [15] or because patients are attending an unfamiliar environment [16], a possibility apparently confirmed in previous studies [7,12-14].

Misclassification of hypertensive patients due to the influence of a 'white-coat hypertension' might indeed result in overtreatment of patients at relatively limited risk. However, the data obtained from large cohorts seen by general practitioners in Italy $[3,4]$ and the lower BP thresholds for hypertension diagnosis recommended for ABP data [8] seem rather to suggest the occurrence of an opposite phenomenon, that is, a high number of hypertensive patients being inadequately managed because of insufficiently aggressive protocols, thus remaining at high risk of cardiovascular and cerebrovascular accidents.

We therefore deemed it appropriate to experimentally investigate, in Italian practices, the extent of agreement between the classification of BP control based on conventional clinic measurements and that derived from ABPM to explore whether a more systematic use of ABPM might be needed for the appropriate management of hypertension by the general practitioner.

\section{Methods}

\section{Participants and data collection}

One hundred ninety general practicioners distributed over the Italian territory agreed to participate in this study. All participating doctors were trained on how to properly perform ABPM and were informed on its value in the management of hypertension through educational courses before entering the study.

Each participating physician recruited a minimum of six consecutive hypertensive patients on stabilized antihypertensive treatment for at least 6 months, who were either regarded as well controlled $(50 \%)$ or not controlled by treatment according to the office BP measurement obtained over the last three visits.

Exclusion criteria were the presence of atrial fibrillation, any severe systemic or psychiatric disease majorly affecting patients' health status, or both, or their inability to reliably perform a 24-h ABP recording.

For each participant who agreed in writing to participate after receiving adequate information, demographic and clinical data were collected (age, sex, date of first diagnosis of hypertension, history of acute coronary syndrome, history of stroke/transient ischemic attack, presence of hypercholesterolaemia, hypertriglyceridaemia, diabetes mellitus, and peripheral arterial disease, as recorded in the practice file; BMI, waist circumference, alcohol consumption, and smoking habits recorded at visit). Office $\mathrm{BP}$ according to the usual practice procedures was recorded at the time of the visit in the seated position, together with information on the current antihypertensive therapy and on any other therapy prescribed. As mentioned above, the patient was then classified by the attending physician as having a 'controlled' or 'noncontrolled' hypertension based on the average of the BP values obtained during last three doctor's visits.

Subsequently, each patient performed a 24-h ABPM, making use of the validated A\&D TM 2430 device [11]. Measurements were programmed to be taken every $15 \mathrm{~min}$ during daytime and every $30 \mathrm{~min}$ during the night-time. The ABPM device was applied in the physician's office. The ABPM file, as well as the file including clinical data, was anonymously transferred by each doctor to a Core center hosting the study database, using a validated secure proprietary internet technique (Hypernet).

\section{Data analysis}

Data were analysed by an independent CRO, Hyperphar Group (Milan, Italy), using SPSS, version 14, for Windows (SPSS Inc., Chicago, Illinois, USA), after checking the quality of each ABP recording according to the requirements issued by the Italian Society of Hypertension [17] and by the European Society of Hypertension working group on BP monitoring [9]. Patients were classified as having ABP controlled based on the current guidelines (average of the daytime awake period $<135$ / $85 \mathrm{mmHg}$ and average of nocturnal sleep time $<120 /$ $70 \mathrm{mmHg}$ ). Daytime and night-time periods were defined based on wide fix intervals $(07: 00-22: 00 \mathrm{~h}$, daytime; 22:00-07:00 $\mathrm{h}$ night-time). ABP values higher than suggested thresholds were taken as to indicate uncontrolled ABP [8]. Usual descriptive statistics were used to analyse the study data; comparisons between subgroups were performed with the chi square test (polychotomous nominal), Cochran-Mantel-Haenszel test of the equality of odds ratios (dichotomous nominal), Mann-Whitney $U$-test (continuous variables with nonhomogeneous 
Table 1 Demographic and lifestyle profile of the sample

\begin{tabular}{|c|c|c|c|c|}
\hline Variable & $\begin{array}{c}\text { Hypertension } \\
\text { controlled }(N=894)\end{array}$ & $\begin{array}{c}\text { Hypertension not } \\
\text { controlled }(N=874)\end{array}$ & Total $(N=1768)$ & Statistics \\
\hline Sex, $n$ women (\%) & $453(50.7)$ & $472(54.0)$ & $925(52.3)$ & $0.161^{\mathrm{g}}$ \\
\hline Ethnicity, $n$ white $(\%)^{\mathrm{a}}$ & $889(99.4)$ & $866(99.1)$ & $1755(99.3)$ & $0.386^{9}$ \\
\hline Age (years), mean \pm SD [range] & $60.6 \pm 11.8[19-93]$ & $61.5 \pm 12.2[26-98]$ & $61.1 \pm 12.0[19-98]$ & $0.120^{h}$ \\
\hline Duration of hypertension (months), mean $\pm \mathrm{SD}$ [range] & $84.5 \pm 68.3[6-485]$ & $92.6 \pm 74.3[6-543]$ & $88.5 \pm 71.4[6-543]$ & $0.033^{i}$ \\
\hline BMI $\left(\mathrm{kg} / \mathrm{m}^{2}\right)$, mean \pm SD range] $^{\mathrm{b}}$ & $27.4 \pm 4.0[14.6-44.4]^{\mathrm{e}}$ & $28.1 \pm 4.5[16.4-47.7]^{f}$ & $27.7 \pm 4.3[14.6-47.7]$ & $<0.001^{\mathrm{i}}$ \\
\hline Obesity, $n$ obese $(\%)^{\mathrm{b}}$ & $208(23.7)^{\mathrm{e}}$ & $258(30.4)^{f}$ & $466(27.0)$ & $0.002^{9}$ \\
\hline At risk from waist circumference ${ }^{c}$ & $367(48.1)$ & $414(56.4)$ & $781(52.2)$ & $0.001^{9}$ \\
\hline Active smoking, $n(\%)^{d}$ & $120(13.3)$ & $136(15.8)$ & $256(14.7)$ & $0.197^{9}$ \\
\hline Alcohol consumption, $>2 \mathrm{U} /$ day, $n(\%)^{\mathrm{e}}$ & $38(4.4)$ & $50(5.9)$ & $88(5.1)$ & $0.141^{9}$ \\
\hline Nonantihypertensive therapy, $n(\%)$ & $424(47.4)$ & $452(51.7)$ & $876(49.5)$ & $0.071^{\mathrm{g}}$ \\
\hline Number of nonantihypertensive drugs, mean $\pm S D$ [range $]^{f}$ & $1.9 \pm 1.2[1-8]$ & $1.9 \pm 1.2[1-9]$ & $1.9 \pm 1.2[1-9]$ & $0.809^{h}$ \\
\hline
\end{tabular}

${ }^{a}$ Others, including two Asiatic, two African, nine Hispanic. ${ }^{\mathrm{b}}$ Obese: $>30.0 \mathrm{~kg} / \mathrm{m}^{2} ; 17$ controlled missing, 23 noncontrolled missing. ${ }^{\mathrm{c}}$ At risk: $>88 \mathrm{~cm}$ if woman, $>102 \mathrm{~cm}$ if man; 133 controlled missing, 140 noncontrolled missing. ${ }^{\mathrm{d}}$ Fourteen controlled missing, 16 noncontrolled missing. ${ }^{\mathrm{e}}$ Twenty-two controlled missing, 31 noncontrolled missing. ${ }^{f}$ Only among those taking at least one; most frequent: serum lipid reducing agents (19.6\% of patients), antithrombotic agents (18.2\%), and drugs used in diabetes (12.7\%). ${ }^{\mathrm{g}}$ Cochran-Mantel-Haenszel test for the equality of the odds ratio. ${ }^{\mathrm{h}}$ Unpaired $t$-test not assuming equality of variances. ${ }^{\mathrm{i}} \mathrm{Mann}-\mathrm{Whitney} U$-test.

variances), and $t$-test (continuous variables with homogeneous variances). The analyses of agreement between techniques were performed using $\kappa$ statistics; positive and negative predictive values (PPV and NPV) were computed using the classification based on ABPM as reference standard and office BP measurement as test technique. All reported $P$ values are two-tailed and have descriptive value only.

The sample size was set at approximately 2000 patients, with 1500 valid ABPM recordings being required to estimate with a power of $95 \%$ and a $\beta$ value of less than 0.05 , with a degree of discordance between measures of $1 \%$ or more.

The study was performed in compliance with the Declaration of Helsinki, and the protocol, information, and consent procedures were approved by the Ethics Committees of the participating institutions. Participating doctors were trained and certified by the Italian Society of General Practice and the European School of General Practice. The study Steering Committee included the authors of this paper, and was responsible for the scientific reliability of the procedures.

\section{Results}

Between 1 July 2005 and 27 November 2006, 190 practicioners recruited 2059 patients: 27 physicians recruited four or less patients, 124 recruited 5-10 patients, and 39 recruited at least 11 patients. Overall, data of 291 patients were excluded from analysis because these patients were unable to provide all data required by the protocol (14 did perform ABPM and were considered in the safety section). ABPM was performed in 1768 patients, with a valid 24-h ABP profile being obtained in 1524 of them.

Among the 1768 patients included, 894 (50.6\%) were reported as 'controlled'; the remaining ones as 'not controlled' based on office BP. Table 1 summarizes the demographic and lifestyle profile of these patients and Table 2 summarizes their medical history and antihypertensive medications. As expected, patients with noncontrolled hypertension included individuals with

Table 2 Medical history and antihypertensive medications

\begin{tabular}{|c|c|c|c|c|}
\hline & $\begin{array}{l}\text { Hypertension controlled } \\
\qquad(n=894)\end{array}$ & $\begin{array}{l}\text { Hypertension not controlled } \\
\qquad(n=874)\end{array}$ & Total $(n=1768)$ & Statistics \\
\hline History of acute coronary syndrome, $n(\%)$ & $64(7.2)$ & $66 / 874(7.6)$ & $130(7.4)$ & $0.752^{d}$ \\
\hline History of stroke/TIA, $n(\%)$ & $40(4.5)$ & $38 / 874(4.3)$ & $78(4.4)$ & $0.897^{d}$ \\
\hline Hypercholesterolaemia, $n(\%)$ & $305(34.5)^{\mathrm{a}}$ & $352(40.4)^{b}$ & $660(37.4)$ & $0.011^{d}$ \\
\hline Hypertriglyceridaemia, $n(\%)$ & $131(14.7)^{b}$ & $178(20.4)^{b}$ & $309(17.5)$ & $0.002^{d}$ \\
\hline PAD, $n(\%)$ & $20(2.2)^{\mathrm{c}}$ & $34(3.9)^{\mathrm{a}}$ & $54(3.0)$ & $0.046^{d}$ \\
\hline Diabetes, $n(\%)$ & $101(11.3)$ & $145(16.6)$ & $246(13.9)$ & $0.001^{d}$ \\
\hline Diuretics, $n(\%)$ & $380(42.5)$ & $428(49.0)$ & $808(45.7)$ & $0.006^{d}$ \\
\hline ACE-inhibitors, $n(\%)$ & $409(45.7)$ & $388(44.4)$ & $797(45.1)$ & $0.567^{d}$ \\
\hline Beta-blockers, $n(\%)$ & $264(29.5)$ & $299(34.2)$ & $563(31.8)$ & $0.035^{d}$ \\
\hline AT-II blockers, $n(\%)$ & $257(28.7)$ & $289(33.1)$ & $546(30.9)$ & $0.050^{d}$ \\
\hline $\mathrm{Ca}$ antagonists, $n(\%)$ & $228(25.5)$ & $304(34.8)$ & $532(30.1)$ & $<0.001^{\mathrm{d}}$ \\
\hline Alpha-blockers, $n(\%)$ & $76(8.5)$ & $93(10.6)$ & $169(9.6)$ & $0.127^{d}$ \\
\hline Therapeutic classes in use, mean \pm SD [range] & $1.81 \pm 0.86[1-5]$ & $2.06 \pm 1.01[1-6]$ & $1.93 \pm 0.94[1-6]$ & $<0.001^{\mathrm{e}}$ \\
\hline Total daily units, mean \pm SD [range] & $1.90 \pm 0.96[1-6]$ & $2.17 \pm 1.11[1-7]$ & $2.03 \pm 1.05[1-7]$ & $<0.001^{\mathrm{e}}$ \\
\hline Last office SBP recorded, mean \pm SD [range] & $137.0 \pm 13.3[97-190]$ & $151.2 \pm 14.9[80-210]$ & $144.0 \pm 15.8[80-210]$ & $<0.001^{\mathrm{f}}$ \\
\hline Last office DBP recorded, mean \pm SD [range] & $82.4 \pm 8.5[50-118]$ & $89.2 \pm 9.5[50-140]$ & $85.8 \pm 9.6[50-140]$ & $<0.001^{f}$ \\
\hline Last office HR recorded, mean $\pm \mathrm{SD}$ [range] & $73.7 \pm 9.3[47-115]$ & $75.4 \pm 9.5[50-120]$ & $74.6 \pm 9.5[50-120]$ & $0.001^{f}$ \\
\hline
\end{tabular}

ACE, angiotensin-converting enzyme; AT, anti-thrombin; HR, heart rate; PAD, peripheral arterial disease; TIA, transient ischemic attack. ${ }^{a}$ Two missing information. ${ }^{b}$ Three missing information. ${ }^{\mathrm{c}}$ One missing information. ${ }^{\mathrm{d}}$ Cochran-Mantel-Haenszel test for the equality of the odds ratio. ${ }^{\mathrm{e}}$ Mann-Whitney $U$-test. ${ }^{f} t$-test. 
Table 3 Ambulatory blood pressure monitoring data

\begin{tabular}{|c|c|c|c|c|}
\hline & Controlled $(n=763)$ & Noncontrolled $(n=761)$ & Total $(n=1524)$ & Statistics \\
\hline Mean 24-h SBP & $132.7 \pm 12.8[101.7-230.3]$ & $139.5 \pm 14.3[104.2-225.2]$ & $136.1 \pm 14.0[101.7-230.3]$ & $<0.001^{\mathrm{c}}$ \\
\hline Mean 24-h DBP & $78.2 \pm 6.9[57.2-102.0]$ & $80.8 \pm 8.5[60.4-110.9]$ & $79.5 \pm 7.9[57.2-110.9]$ & $<0.001^{\mathrm{c}}$ \\
\hline Mean 24-h HR & $71.7 \pm 8.5[47.0-101.7]$ & $71.6 \pm 8.8[49.2-102.9$ & $71.6 \pm 8.7[47.0-102.9]$ & $0.786^{\mathrm{b}}$ \\
\hline Mean daytime SBP & $136.0 \pm 13.0[103.2-223.8]$ & $142.8 \pm 14.7[103.1-241.9]$ & $139.4 \pm 14.3[103.1-241.9]$ & $<0.001^{\mathrm{c}}$ \\
\hline Mean daytime DBP & $80.4 \pm 7.6[59.0-106.6]$ & $83.2 \pm 9.2[60.1-111.4]$ & $81.8 \pm 8.6[59.0-111.4]$ & $<0.001^{c}$ \\
\hline Mean daytime HR & $74.5 \pm 9.2[49.2-107.6]$ & $74.2 \pm 9.4[51.6-107.4]$ & $74.3 \pm 9.3[49.2-107.6]$ & $0.471^{\mathrm{b}}$ \\
\hline Mean night-time SBP & $121.0 \pm 17.7[82.4-226.3]$ & $127.4 \pm 19.1[85.5-220.4]$ & $124.2 \pm 18.7[82.4-226.3]$ & $<0.001^{\mathrm{c}}$ \\
\hline Mean night-time DBP & $70.1 \pm 9.3[48.0-108.0]$ & $72.4 \pm 10.1[48.1-107.8]$ & $71.2 \pm 9.7[48.0-108.0]$ & $<0.001^{c}$ \\
\hline Mean night-time HR & $62.3 \pm 8.6[38.5-98.5]$ & $62.6 \pm 9.2[36.3-99.9]$ & $62.4 \pm 8.9[36.3-99.9]$ & $0.416^{b}$ \\
\hline Nocturnal SBP drop ${ }^{a}$ & $15.0 \pm 14.7[-55.1$ to +69.2$]$ & $15.5 \pm 16.3[-66.3$ to +65.0$]$ & $15.2 \pm 15.5[-66.3$ to +69.2$]$ & $0.463^{\mathrm{c}}$ \\
\hline Nocturnal DBP drop ${ }^{a}$ & $10.3 \pm 9.1[-18.9$ to +38.8$]$ & $10.9 \pm 9.4[-34.6$ to +44.2$]$ & $10.6 \pm 9.2[-34.6$ to +44.2$]$ & $0.229^{b}$ \\
\hline Nocturnal HR drop ${ }^{\mathrm{a}}$ & $12.3 \pm 7.3[-14.4$ to +37.3$]$ & $11.6 \pm 7.8[-18.9$ to +35.1$]$ & $11.9 \pm 7.5[-18.9$ to +37.3$]$ & $0.063^{\mathrm{b}}$ \\
\hline
\end{tabular}

Data are given in mean \pm SD [range]. HR, heart rate. ${ }^{a}$ Difference between mean daytime average and mean night-time average; negative means an increase. ${ }^{b}$ Unpaired $t$-test not assuming equality of variances. ${ }^{\mathrm{C}} \mathrm{Mann}-$ Whitney $U$-test.

significantly greater body weight and waist circumference than those with controlled hypertension. Furthermore, they had a significantly longer history of hypertension; higher proportion of concurrent diseases, especially diabetes; and were receiving significantly more antihypertensive agents (especially, diuretics, $\beta$-blockers, and $\mathrm{Ca}$ antagonists).

In 244 patients, the 24-h ABP recording was rated as not valid. High-quality ABPM in a general practice setting could thus be obtained in 1524 out of 1768 patients in a per protocol analysis or in 1524 out of 1970 patients according to an intention-to-treat approach, by including patients who should have performed ABPM (seen at the office, no recorded exclusion criteria) but who did not. The feasibility of ABPM in general practice setting can, therefore, be estimated as ranging between $77.4 \%$ [95\% confidence interval $(\mathrm{CI})=75.4-79.2]$ and $86.2 \%(95 \%$ $\mathrm{CI}=84.5-87.8)$. The proportion of valid ABP recordings was similar between controlled $(85.3 \%$; $95 \% \mathrm{CI}=82.8-$
87.6) and noncontrolled patients $(87.1 \%$; $95 \% \mathrm{CI}=84.6-$ $89.2 \% ; \quad P=0.294$, Cochran-Mantel-Haenszel test). Overall, only 332 out of 1524 valid ABP recordings identified a 'controlled' hypertension over $24 \mathrm{~h}$. Out of the 1192 recordings 'not controlled' for ABP, 749 (62.8\%) were not controlled during both day and night; 226 (19.0\%) were not controlled during the night but controlled during the day, and $217(18.2 \%)$ were not controlled during the day but controlled during the night (data not shown). Table 3 summarizes the ABPM data.

The agreement between office and ABPM classification of BP control was overall poor $(\kappa=0.120$; Figs 1 and 2$)$. A patients defined as 'not controlled' during the visit by the attending physician had more than $80 \%$ probability to be found 'not controlled' also by 24-h ABPM. On the contrary, a patient defined as 'controlled' by the attending physician had more than $70 \%$ probability to be found 'not controlled' by ABPM. Among diabetic patients, the latter proportion increased to almost $75 \%$ by considering the

Fig. 1

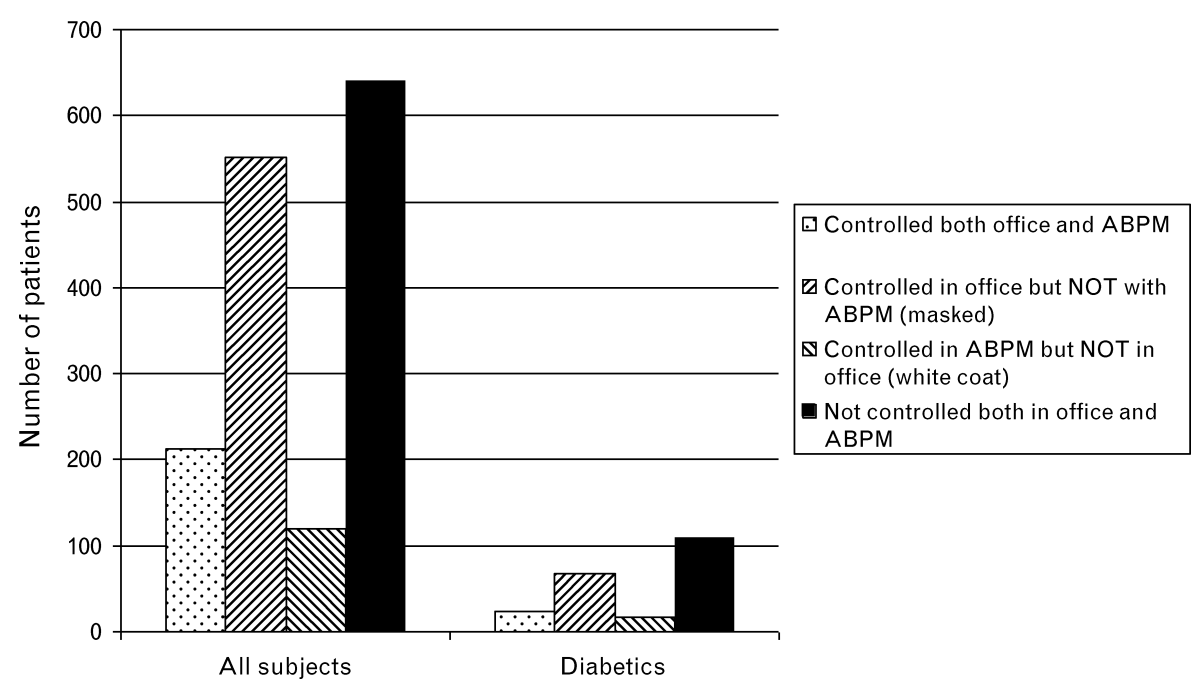

Agreement (all patients) between office and ambulatory blood pressure monitoring classification of blood pressure control. ABPM, ambulatory blood pressure monitoring. 


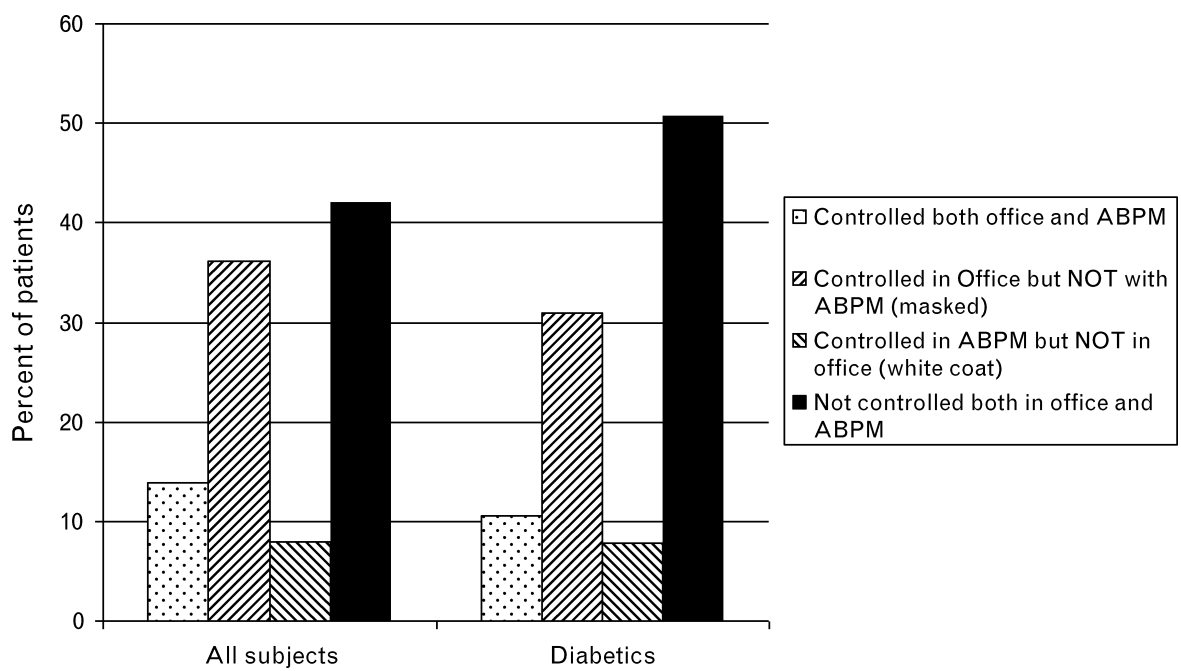

Agreement (percent) between office and ambulatory blood pressure monitoring classification of blood pressure control. ABPM, ambulatory blood pressure monitoring.

same 24-h ABP normalcy cut-off value as in nondiabetic patients, in absence of more precise guidelines.

Taking 24-h ABP as reference standard, the office measurement had quite a good PPV $(0.842)$ but a poor NPV (0.278). Among diabetic patients, the PPV of the office measurement remained high (0.866) but the NPV decreased to 0.256 .

Specific subgroups of patients, in whom a particularly low NPV of office BP measurements might be expected, were identified with the aim of defining conditions in which it would be appropriate to systematically perform 24-h ABPM. On the basis of the estimates reported in Table 4, performance of 24-h ABPM appeared indicated mostly in patients considered 'controlled' by the general practitioner, of male sex, at least 65 years of age, drinking more than $2 \mathrm{U} /$ day of alcohol, obese, or with diabetes. Among the patients exhibiting three or more among these five characteristics, the PPV of office BP was 0.914 and the NPV was 0.149. The patients in this subgroups represented approximately $12 \%$ of the monitored population of hypertensive patients on treatment.

\section{Safety}

In the present study, 72 patients out of the 1842 who performed ABPM (of whom 1768 analysed and 74 protocol violators) prematurely interrupted the recording $(4.0 \%$; $95 \% \mathrm{CI}=3.1-5.0)$, in 51 of them because of intolerance or discomfort, in 17 because of technical problems, and in four because of refusal to complete the 24-h recording. The total number of undesired reactions, including those not ending in premature test interruption, amounted to 224 events in 204 patients (11.1\%; $95 \% \mathrm{CI}=9.7-12.6$ ).
These events included effects of the compressive action of the cuff (expressed as pain, local discomfort, topical reactions from swelling to rash, local haematomas/ecchymoses, and hand paraesthesias: 163 events), disturbances during sleep (54 events), and anxiety or general discomfort associated with the procedure (seven events). The intensity was reported as mild for 120 events, moderate

Table 4 Positive and negative predictive value of office blood pressure measurement by subgroup

\begin{tabular}{|c|c|c|c|}
\hline Factor & Level & PPV & NPV \\
\hline All patients & & 0.482 & 0.278 \\
\hline \multirow[t]{3}{*}{ Age (years) } & $<55$ & 0.833 & 0.290 \\
\hline & $55-64$ & 0.845 & 0.304 \\
\hline & $\geq 65$ & 0.847 & 0.248 \\
\hline \multirow[t]{2}{*}{ Active smokers } & No & 0.850 & 0.286 \\
\hline & Yes & 0.800 & 0.260 \\
\hline \multirow[t]{2}{*}{ Alcohol abusers } & No & 0.842 & 0.282 \\
\hline & Yes & 0.911 & 0.097 \\
\hline \multirow[t]{2}{*}{ Sex } & Men & 0.901 & 0.243 \\
\hline & Women & 0.788 & 0.315 \\
\hline \multirow[t]{2}{*}{ Hypercholesterolaemia } & No & 0.860 & 0.254 \\
\hline & Yes & 0.816 & 0.324 \\
\hline \multirow[t]{2}{*}{ Hypertriglyceridaemia } & No & 0.829 & 0.280 \\
\hline & Yes & 0.892 & 0.275 \\
\hline \multirow[t]{2}{*}{ History of CHD } & No & 0.839 & 0.278 \\
\hline & Yes & 0.877 & 0.271 \\
\hline \multirow[t]{2}{*}{ History of stroke/TIA } & No & 0.841 & 0.277 \\
\hline & Yes & 0.871 & 0.294 \\
\hline \multirow[t]{2}{*}{ PAD } & No & 0.838 & 0.273 \\
\hline & Yes & 0.966 & 0.500 \\
\hline \multirow[t]{2}{*}{ Diabetes } & No & 0.838 & 0.281 \\
\hline & Yes & 0.866 & 0.256 \\
\hline \multirow[t]{2}{*}{ Obese } & No & 0.834 & 0.288 \\
\hline & Yes & 0.865 & 0.251 \\
\hline \multirow[t]{2}{*}{ Risk from abdominal fat } & No & 0.853 & 0.268 \\
\hline & Yes & 0.840 & 0.289 \\
\hline \multirow[t]{2}{*}{ Antihypertensive drugs } & $1-2$ & 0.853 & 0.266 \\
\hline & 3 or more & 0.819 & 0.321 \\
\hline
\end{tabular}

CHD, coronary heart disease; NPV, negative predictive value; PAD, peripheral arterial disease; PPV, positive predictive value; TIA, transient ischemic attack. 
for 80 , and severe for 17 . The 17 reactions classified as severe led to premature cuff removal by the patients (except in three patients) and included pain at compression (in seven), sleep disturbances (in four), topical reactions (in two), hand paraesthesias (in two), and anxiety and discomfort (in one each). Corrective actions by the physician (usually consisting in repositioning of the cuff) were rarely taken because the onset of the above reaction was normally not immediate.

\section{Discussion}

This study is, to our knowledge, the first nationwide evaluation of the concordance between the definition of hypertension control by general practitioners' based on conventional BP measurements and the corresponding definition based on data obtained from 24-h ABPM performed in the same patients. The main result of our study is that patients found not to be controlled by conventional BP measurements at the general practitioner's office were likely to be found so also by 24-h ABPM, whereas patients found controlled by office BP measurements were frequently found not controlled according to ABPM. This discrepancy does not carry only methodological implications but it is also of substantial clinical relevance among high-risk patients who need accurate management of their hypertension.

On theoretical grounds, these findings may lead to the conclusion that hypertensive patients found 'controlled' in the office under antihypertensive treatment should be considered for 24-h ABPM performance by the general practitioner based on the expectation that approximately $66 \%$ of the hypertensive population may be found 'not controlled' according to ABPM criteria. This theoretical systematic approach, however, would result in the prescription of a very high number of 24-h ABP recordings with a significant impact on healthcare costs, also taking into account the increasing proportion of hypertensive patients in the population. The findings of our study may offer a practical solution to this difficulty, however. On the basis of the search of possible determinants of the discrepancy between the identification of hypertension control provided by office and ambulatory BP measurements, our data allow the identification of relatively small subgroups of hypertensive patients in whom 24h ABPM should indeed be performed, aimed at effectively reducing cardiovascular risk. Among hypertensive patients considered as 'controlled' by the general practitioner during the office visit, patients of male sex, aged at least 65 years, alcohol consumers ( $>2 \mathrm{U} / \mathrm{day})$, with obesity and diabetes would in particular need 24-h ABPM to be performed. This suggestion is based on the fact that, among the patients carrying three or more of these five features, the PPV of office BP, in identifying controlled 24-h ABP values, was 0.914, whereas the NPV was only 0.149 . The patients in this subgroup represented approximately $12 \%$ of the population of hypertensive patients on treatment included in our study.

An additional finding of our study is related to ABPM application by general practitioners, who appear able to obtain valid 24-h ABP recordings in $85 \%$ of hypertensive patients. This performance is not too different from that reported in specialized centres [9]. It has to be acknowledged, however, that this result may not faithfully reflect the performance of the 'average' general practitioner in Italy because doctors participating in this study underwent specific training, which is not usually accessible to all general practitioners.

A direct comparison with previous similar experiences in this regard can hardly be made because of the limited number of studies available on this issue and due to the change in ABPM evaluation criteria introduced by recent ABPM guidelines $[8,9,17]$. In a much smaller cohort in Ireland (381 patients), 33.8\% had a normal BP result on ABPM [18] compared to the $21.8 \%$ seen in the present study, a finding that may be explained by methodological differences. Several studies [7,12-14] have been performed to explore the need to reduce treatment intensity on the assumption that office measurements might be affected by a substantial 'white-coat effect'. This condition (i.e. 'noncontrolled' patients in the office found controlled at 24-h ABPM), however, was observed only in $7.9 \%$ of patients included in our study, a finding not substantially different from the data reported in the literature in a similar setting [19]. Thus, our study suggests that the condition referred to as "white-coat hypertension' is infrequently found in general practice, probably because of the relationship between patients and their family doctor is significantly different than that between patients and doctors working in a specialist clinic. Conversely, a 'masked hypertension' condition is more frequently identified in this setting, which emphasizes the need to obtain information on out-ofoffice BP in a relatively high number of patients. This could be done either through 24-h ABPM performance or through a more frequent implementation of home $\mathrm{BP}$ monitoring [20]. Our study was not designed to explore this issue, but the similarities and differences between the information on out-of-office BP provided by home and ABPM is a topic of great interest [21-23], which would, however, require further investigation in future studies.

\section{Conclusion}

Our study emphasizes the importance of PPV of office readings in identifying patients with uncontrolled 24-h $\mathrm{BP}$ in general practice. Our data, however, also emphasize that the finding of controlled office BP in this setting should be taken with caution because it might not faithfully reflect 24-h BP control in daily life. [24] This calls for a larger use of 24-h ABPM also in clinical practice, and our study offers some indication on the clinical features 
that might help identifying those patients in whom implementation of this approach can be particularly useful and may allow a more efficient reduction of BPrelated patient's cardiovascular risk.

\section{Acknowledgements}

This study had been sponsored initially by GlaxoSmithKline, Verona, Italy, then by Bayer-Schering Pharma, Milan, Italy, which covered the costs for setup, management, and analysis of the study, and the renting of the ABPM devices.

Ethics approval was issued by each Ethics Committee competent for the territory where the practices are located, in compliance with the Italian regulations.

There are no conflicts of interest.

The authors wish to thank all participating general practitioners (listed in the appendix) and all the participating patients.

\section{References}

1 Mancia G, De Backer G, Dominiczak A, Cifkova R, Fagard R, Germano G, et al. 2007 Guidelines for the Management of Arterial Hypertension: the Task Force for the Management of Arterial Hypertension of the European Society of Hypertension (ESH) and of the European Society of Cardiology (ESC). J Hypertens 2007; 25:1105-1187.

2 Gensini GF, Zaninelli A, editors. The Italian Guidelines on Stroke, Release of February 2007. http://old.spread.it/SpreadEng/EnglHome.htm. [Accessed 22 December 2007]

3 Filippi A, Vanuzzo D, Bignamini AA, Mazzaglia G, Brignoli O, Sabatini A, et al. Secondary prevention of myocardial infarction: a survey in primary care. J Cardiovasc Med (Hagerstown) 2006; 7:422-426.

4 Filippi A, Bignamini AA, Sessa E, Samani F, Mazzaglia G. Secondary prevention of stroke in Italy: a cross-sectional survey in family practice. Stroke 2003; 34:1010-1014.

5 Zaninelli A, Cofrancesco E, Parati G, Filippi A, Francucci B, Agabiti-Rosei E, et al. Hypertension assessment and management in general practice. The SHYGMO project. Am J Hypertens 2001; 14:138A.

6 Mancia G, Parati G. Office compared with ambulatory blood pressure in assessing response to antihypertensive treatment: a meta-analysis. J Hypertens 2004; 22:435-445.

7 Marchiando RJ, Elston MP. Automated ambulatory blood pressure monitoring: clinical utility in the family practice setting. Am Fam Physician 2003; 67:2343-2350.

8 O'Brien E, Asmar R, Beilin L, Imai Y, Mancia G, Mengden T, et al., European Society of Hypertension Working Group on Blood Pressure Monitoring. Practice guidelines of the European Society of Hypertension for clinic, ambulatory and self blood pressure measurement. $J$ Hypertens 2005; 23:697-701.

9 O'Brien E, Asmar R, Beilin L, Imai Y, Mallion JM, Mancia G, et al., European Society of Hypertension Working Group on Blood Pressure Monitoring. European Society of Hypertension recommendations for conventional, ambulatory and home blood pressure measurement. J Hypertens 2003; 21:821-848.

10 Myers MG, Haynes RB, Rabkin SW. Canadian hypertension society guidelines for ambulatory blood pressure monitoring. Am J Hypertens 1999; 12 (11 Pt 1):1149-1157.

11 Pickering T. Recommendations for the use of home (self) and ambulatory blood pressure monitoring. American Society of Hypertension Ad Hoc Panel. Am J Hypertens 1996; 9:1-11.

12 Ragot S, Sosner P, Yau C, Brunel P, Herpin D. Management in general practice of hypertensive patients poorly controlled with a fixed-dose reninangiotensin system inhibitor and diuretic combination: results from a French national survey. J Hum Hypertens 2006; 20:407-418.

13 Lorgelly P, Siatis I, Brooks A, Slinn B, Millar-Craig MW, Donnelly R, Manning G. Is ambulatory blood pressure monitoring cost-effective in the routine surveillance of treated hypertensive patients in primary care? $\mathrm{Br} J$ Gen Pract 2003; 53:794-796.

14 Brown MA, Buddle ML, Martin A. Is resistant hypertension really resistant? Am J Hypertens 2001; 14:1263-1269.
15 Mancia G, Sega R, Bravi C, De Vito G, Valagussa F, Cesana G, Zanchetti A. Ambulatory blood pressure normality: results from the PAMELA study. $J$ Hypertens 1995; 13 (12 Pt 1):1377-1390.

16 Zaninelli A, Boni E, Fariello R, Alicandri C, Muiesan G. Variability of blood pressure measured in the physician's office and at patient's domicile [in Italian]. Cardiologia 1988; 33:87-92.

17 Parati G, Omboni P, Palatini P, Rizzoni D, Bilo G, Valentini M, et al., on behalf of the Italian Society of Hypertension Working Group on Blood Pressure Monitoring. Italian Society of Hypertension Guidelines for conventional and automated blood pressure measurements in the office, at home and over 24 h. High Blood Press Cardiovasc Prev 2008; 15:283310.

18 Uallachain GN, Murphy G, Avalos G. The RAMBLER study: the role of ambulatory blood pressure measurement in routine clinical practice - a cross-sectional study. Ir Med J 2006; 99:276-279.

19 Verberk WJ, Kroon AA, Thien T, Lenders JW, van Montfrans GA, Smit AJ, de Leeuw PW. Prevalence of the white-coat effect at multiple visits before and during treatment. J Hypertens 2006; 24:2357-2363.

20 Parati G, Stergiou GS, Asmar R, Bilo G, de Leeuw P, Imai Y, et al., on behalf of the ESH Working Group on Blood Pressure Monitoring. European Society of Hypertension guidelines for blood pressure monitoring at home: a summary, Report of the Second International Consensus Conference on Home Blood Pressure Monitoring. J Hypertens 2008; 26:1505-1530.

21 Parati G, Pickering TG. Home blood-pressure monitoring: US and European consensus. Lancet 2009; 373:876-878.

22 Parati G, Omboni S, Bilo G. Why is out-of-office blood pressure measurement needed? Home blood pressure measurements will increasingly replace ambulatory blood pressure monitoring in the diagnosis and management of hypertension. Hypertension 2009; 54:181 187.

23 Verdecchia P, Angeli F, Mazzotta G, Gentile G, Reboldi G. Home blood pressure measurements will not replace 24-hour ambulatory blood pressure monitoring. Hypertension 2009; 54:188-195.

24 Rastas S, Pirttila T, Viramo P, Verkkoniemi A, Halonen P, Juva K, et al. Association between blood pressure and survival over 9 years in a general population aged 85 and older. J Am Geriatr Soc 2006; 54:912-918.

\section{Appendix}

List of participating general practitioners: Abbate Giuseppe, Agati Riccardo, Alborghetti Ivan, Allasia Bartolomeo, Ammannati Francesco, Antico Romano, Argena Antonio, Baglioni Gregorio, Barone Giovanni, Bellini Felice, Bitetti Rocco, Boncompagni Salvatore, Bonvincini Angelo, Borchi Remo, Boschi Giuseppe, Brancati Ciro, Bressani Anna Maria, Brunelli Brunello, Bruno Lucio Angelo, Bruschelli Carla, Bucigno Fausto, Bufano Carmine, Caccamo Orazio Antonio, Caiello Paola, Calabria Elio, Calcini Silvio, Caligiore Giuseppe, Calzolari Mauro, Campolongo Domenica, Canizzo Diego, Cannelli Bernardo, Cantini Andrea, Capotorto Giuseppe, Caprioli Giuseppe, Carducci Alberto, Caruso Marco, Casarola Sergio, Cecchi Massimo, Ciccone Roberto, Cicconofri Beatrice, Cimignoli Emanuela, Ciotola Pietro, Coletta David, Colomasi Giuseppe, Colosa Giuseppe, Colpani Camilla, Comina Aldo, Condello Domenico, Coppini Bruno, Coppotelli Alberto, Corallo Giovanni, Corrado Carnemolia, Cosima Musci, Crapanzano Andrea, Croce Giuseppe, Curreri Antonio, Dainelli Giuseppe, D'Ambrosio Gaetano, D'Amico Giuseppe, De Bellis Giovanni, De Simone Mario, Dell'Orco Mario Domenico, Dezio Giovanni, Di Giacomo Giovanni, Di Guardo Antonino, Di Natale Massimo, Diaco Goffredo, Dibitetto Nicola, Dolcetti Umberto, Draghini Leonardo, Duranti Giuliana, D'Urso Giuseppe, Ercolino Luigi, Fazio Francesco, Ferrante Tullio, Ferraro Salvatore, Filetti 
Giuseppe, Filippi Alessandro, Filippini Giovanni, Finessi Riccardo, Fiorentini Guido, Fiumana Marzio, Fogher Michele, Fontana Francesco, Foppa Luciano, Gabriele Pino, Gadaleta Caldarola Gennaro, Gangi Fabrizio, Gasparin Amedeo, Gazzurra Stefano, Gerace Antonio, Germano Francesco, Germini Fabrizio, Ghezzi Pietro, Giacci Luciano, Gianderico Alberto, Giannone Alfonso, Giardina Giovanni, Graffigna Renato, Grazzini Marcello, Greco Antonietta, Guerra Antonio, Guida Angelica, Iaccarino Patrizia, Ianiro Gabriella, Intorre Luigi, La Greca Giuseppe, La Vecchia Diego, Lamonica Calogero, Laringe Matteo, Leoni Vincenzo, Lo Vullo Antonino, Loci Antonio, Losacco Renato, Maestoso Carmine, Magi Lorenzo, Manlio Judica, Manzoni Giuseppe, Marangio Giovanni, Mascaretti Claudio, Mastriforti Liliana, Maurici Vincenzo, Melani Paolo, Michelini Vittorino, Micillo Enzo Bruno, Milani Monica, Minervino Francesco, Moniaci Felice, Morgana Ignazio, Muratore Angelo, Murolo Nicola G.M., Musso Paolo, Napoli Luigi, Natali
Roberto, Natalini Bruno, Nati Giulio, Negri Fabrizio, Nicoli Sergio, Nista Nunzia, Paci Carmelo, Paganelli Libero, Pantaleone Caliandro, Paolini Italo, Papi Giancarlo, Parretti Damiano, Parrini Andrea, Pasculli Domenico, Pasinetti Mariella, Pasqualetto Salvatore, Penna Antonio, Pericoli Roberto, Perrone Angela, Piccinocchi Gaetano, Piccolo Francesco, Piccolo Luigi, Piras Enrico, Pomponi Angela, Portas Gianfranco, Posella Raffaele, Roberto Zelante, Rossi Alessandro, Rossi Sergio, Rossi Carmelo Luciano, Rubicini Giuseppe, Sajeva Giuseppina, Salvio Giuliano, Samani Fabio, Sandullo Antonino, Scotti Aldo, Scrofani Angela, Sergio Claudio, Sessa Aurelio, Shafik Kurtam, Sorbo Edgardo, Spadaro Giorgio, Spampinato Alfio, Stramenga Carlo, Surace Maria Antonietta, Taurisano Maria Chiara, Travaglini Rita, Trombatore Alberto, Urbani Alessandro Maria, Vanni Alberto, Varrica Gaetano, Viola Dario, Virgadaula Giorgia, Vitobello Matteo, Zala Massimo, Zaninelli Augusto, Zuccolo Enzo. 